\title{
Amerindian (but not African or European) ancestry is significantly associated with diurnal preference within an admixed Brazilian population
}

Kieren J. Egan ${ }^{1}$, Hadassa Campos Santos ${ }^{2}$, Felipe Beijamini ${ }^{3}$, Nubia E. Duarte ${ }^{2}$, Andréa R.V.R. Horimoto ${ }^{2}$, Tâmara P. Taporoski ${ }^{3}$, Homero Vallada ${ }^{3}$, André B. Negrão ${ }^{2}$, Jose E. Krieger ${ }^{2}$, Mario Pedrazzoli ${ }^{4}$, Kristen L. Knutson ${ }^{5}$, Alexandre C. Pereira ${ }^{2}$, Malcolm von Schantz ${ }^{123}$ *

${ }^{1}$ Faculty of Health and Medical Sciences, University of Surrey, Guildford, Surrey, UK

${ }^{2}$ Laboratory of Genetics and Molecular Cardiology, Heart Institute (Incor), University of

São Paulo Medical School, São Paulo, SP, Brazil

${ }^{3}$ Institute of Psychiatry, University of São Paulo Medical School, São Paulo, SP, Brazil

${ }^{4}$ School of Arts, Sciences, and Humanities, University of São Paulo, São Paulo, SP, Brazil

${ }^{5}$ Department of Medicine, University of Chicago, Chicago, IL Corresponding author

Malcolm von Schantz

Faculty of Health and Medical Science

University of Surrey

Guildford, Surrey, GU2 7XH

United Kingdom

Telephone: +44 1483686468

E-mail: m.von.schantz@surrey.ac.uk 
Abstract

Significant questions remain unanswered regarding the genetic versus environmental contributions to racial/ethnic differences in sleep and circadian rhythms. We addressed this question by investigating the association between diurnal preference, using the Morningness-Eveningness questionnaire (MEQ), and genetic ancestry within the Baependi Heart Study cohort, a highly admixed Brazilian population based in a rural town. Analysis was performed using measures of ancestry, using the Admixture program, and MEQ from 1,453 individuals. We found an association between the degree of Amerindian (but not European of African) ancestry and morningness, equating to 0.16 units for each additional percent of Amerindian ancestry, after adjustment for age, sex, education, and residential zone. To our knowledge, this is the first published report identifying an association between genetic ancestry and $M E Q$, and above all, the first one based on ancestral contributions within individuals living in the same community. This previously unknown ancestral dimension of diurnal preference suggests a stratification between racial/ethnic groups in an as yet unknown number of genetic polymorphisms.

Keywords: Admixture; American Native Continental Ancestry Group; Brazil; Chronotype; Circadian rhythm; Diurnal preference; Sleep homeostasis 


\section{Introduction}

Our species emerged in Africa near the equator, and groups of anatomically modern humans have subsequently colonized regions closer to the poles and the equatorial regions of the other continents. Settlements away from the equator are exposed to higher-amplitude photoperiods, which may have favoured evolutionary adaptions to the circadian system facilitating entrainment across this higher-amplitude photoperiod. Indeed, differences in parameters of the circadian system were observed in the only available report of this kind, a small study comparing African-Americans and EuropeanAmericans, where the former displayed a shorter average circadian period and smaller phase shifts (Eastman, Suh et al., 2015). The same study used ancestrally informative markers (AIMs) to assess degree of genetic admixture. However, previous work has been incapable of adjusting for socio-economic inequalities, which are generally correlated with race/ethnicity, particularly in the United States. A different approach was taken for the current work, where we have used a cohort with a large and well-defined degree of genetic admixture to determine whether genetic ancestry was associated with diurnal preference.

The Baependi Heart Study cohort is based in a small rural town in the state of Minas Gerais in Brazil. (Egan, von Schantz et al., 2016) In this highly admixed population, a mixture of European, African, and Amerindian ancestries is found within virtually all individuals. Here, we aimed to determine whether the degree of each of these ancestries was associated with diurnal preference, based on the morningnesseveningness questionnaire (MEQ) (Horne \& Östberg, 1976). We have previously 
reported an essentially normal distribution of MEQ score within this population, but with a strong general shift towards morningness (von Schantz, Taporoski et al., 2015). The combination of a high degree of admixture, and a largely homogeneous environment and lifestyle, makes this cohort an ideal sample for examining the association between ancestry and circadian preference.

\section{Methods}

The study protocol conformed to the tenets of the Declaration of Helsinki, and was approved by the ethics committee of the Hospital das Clínicas, University of São Paulo. Each subject provided informed written consent before participation. The recruitment and the demographics of this cohort has been described previously. Briefly, this study was set up in 2005 through the recruitment first of randomly selected probands and then of their family members and relatives as far as they could be traced (95 families at baseline). The community is traditional, with a cohesive culture, high degree of admixture, and very limited inbound migration. Collection of diurnal preference/chronotype data using the Brazilian Portuguese version of the MorningnessEveningness questionnaire (Horne \& Östberg, 1976; Benedito-Silva, Menna-Barreto et al, 1989) commenced in April 2013 (von Schantz, Taporoski et al., 2015). Analysis of genomic ancestry was conducted using the Admixture program (Alexander, Novembre et al., 2009), a software tool for maximum likelihood estimation of individual ancestries from multilocus SNP genotype datasets. Specifically, Admixture uses a block relaxation approach to alternately update allele frequency and ancestry fraction genotyped to 
about 900,000 SNPs on Affymetrix (Santa Clara, CA) SNP array 6.0 platform. We assumed as reference populations individuals from the Human Genome Diversity Project (HGDP): Pima, Maya as Amerindians and from the HapMap project, Africans: YRI (Yoruba in Ibadan, Nigeria), LWK (Luhya in Webuye, Kenya), ASW (Americans of African Ancestry in SW USA); European: CEU [Utah Residents (CEPH) with Northern and Western European ancestry] and TSI (Tuscan in Italy).

Analysis was performed using measures of ancestry and MEQ from 1,453 individuals. We used a polygenic mixed model, a well-known methodology for calculating heritability estimates in family studies (de Andrade, Amos et al., 1999).. Here, it was used to quantify the effect of covariates of MEQ score. We used the approach implemented in the kinship2 package in the $\mathrm{R}$ software environment (version 1.5.7, http://CRAN.Rproject.org/package5kinship2) )(Therneau, 2014).

After descriptive analysis, several models were fitted to the data. First, we adjusted three models, European (EUR) African (AFR) and Amerindian (AMR) ancestries separately in each model. Fourth, we adjusted models to test the effect of AMR ancestry with age and sex as covariates, whereas the fifth model also included age, sex, residential zone, and education. Finally, in order to exclude the possibility that the observed associations were the result of floor/ceiling effects for participants over 60 , we repeated the analysis with individuals aged 60 and below.

\section{Results}


The average MEQ score in this sample was $63.5 \pm 10.8$ (average $\pm S D$ ), average age was $46.9 \pm 16.1$ years, $60 \%$ were female, and $87 \%$ were resident in the urban (as opposed to rural) zone of the municipality. Education level within the population $(n=1,453)$ was categorised as follows: $6 \%(90)$ had no schooling, $31.5 \%(458)$ had $1-4$ years of education, $19.8 \%$ (287) had 5-8 years and (390), 27\% 9-11 years. 6.7\% (97) had some further education, and $9 \%$ (131) were university graduates. The ancestral contributions were $78.7 \pm 17.7 \%$ European (range $0.0-99.5 \%$ ), $9.2 \pm 4.5 \%$ Amerindian (range $0.0-28.0 \%)$ ), and $12.1 \pm 18.1 \%$ African (range $0.0-100.0 \%$ ). The distribution of these three ancestries within individuals is shown in Figure 1A. European or African ancestry did not explain a significant proportion of the variation in MEQ score $(\beta=0.020$, $p=0.25$ and $\beta=-0.033, p=0.056$ respectively) whereas there was significant statistical effect for the degree of Amerindian ancestry $(\beta=0.22, p=0.0015)$. For full results, see Table 1. When repeating these analyses to account for the influence of age and sex, a significant association remained for Amerindian ( $\beta$ coefficient $=0.22, p=0.0012$ ). Finally, after the analyses were repeated to account for the influence of age, sex, education, and residential zone, Amerindian ancestry maintained an effect on MEQ (MEQ $\beta=0.16, p=0.009) . \beta$ for other variables were age $\left(0.26, p<1 \times 10^{-5}\right)$, sex $(-1.27$, $p=0.0093)$, education $\left(-0.59, p<1 \times 10^{-5}\right)$ and residence $\left(4.75, p<1 \times 10^{-5}\right)$. Where analyses were repeated with only individuals aged 60 or below, the association between AMR ancestry and MEQ remained significant $(\beta=0.24, p=0.0037)$.

\section{Discussion}


We found that Amerindian, but not African or European ancestry, was associated with greater morningness as assessed through the MEQ within an admixed Brazilian community. To our knowledge, this is the first published report identifying an association between genetic ancestry and MEQ, and above all, the first one based on ancestral contributions within individuals living in the same community.

The MEQ contains two dimensions, a circadian one and one related to sleep homeostasis. ${ }^{7}$ The difference reported here cannot be directly attributed to either of these. It is also possible that differences related to genetic ancestry may relate to circadian period on one hand and to sleep homeostasis in the other in ways which counteract each other in terms of the MEQ. Thus, the fact that we found no significant association between MEQ and African ancestry does not in itself contradict the findings of Eastman and colleagues (Eastman, Suh et al., 2015), which were based on physiological measures related to circadian period, but not sleep homeostasis. It is not inconceivable that adaptation to different latitudes could involve differences in circadian period. For example, an inverse relationship between circadian period length and latitude has been reported in Drosophila (Hut, Paolucci et al., 2013). It is not known whether this relationship also applies in mammals, and indeed, it would not be feasible to investigate this in humans on the scale that would be required.

It has been reported that sleep homeostasis can be influenced by genetic ancestry; a cross-sectional study performed in the United States found greater African ancestry was associated with a lower slow wave sleep to total sleep time ratio in older African Americans (as measured by home sleep recordings) (Halder, Matthews et al., 2015). Within the Baependi cohort, analysis of differences in sleep homeostasis is feasible and 
collection of home sleep recordings, that will eventually allow us to address this issue, have been initiated.

While we endeavoured to make this work as robust as possible, there are a number of limitations, above all unmeasured confounders. Socioeconomic inequalities are controlled for by using the length of education (a close approximation) as a covariate, and by virtue of the fact that Amerindian ancestry is found by varying degrees in the majority of individuals in the sample (as opposed to as a discrete group of individuals). In spite of this, it is never possible to control entirely for socioeconomic inequalities. The association between MEQ and Amerindian ancestry itself is of a modest magnitude 0.22 for each percentage of Amerindian ancestry when adjusted for age and sex, decreasing to 0.16 when adjusted for education and residence as well. By way of comparison, this value is smaller than the average increase in MEQ for each additional year of age (0.26), and yet remained significant when other covariates were added. Our findings represent a previously unknown dimension of diurnal preference influenced by genetic ancestry, suggesting a stratification between different racial/ethnic groups in an unknown number of genetic polymorphisms. Examples of differential distributions of clock gene polymorphisms between such groups have already been reported (Nadkarni, Weale et al., 2005; Dall'Ara, Ghirotto et al., 2016). Thus, whilst this association may contribute to some degree to the generally high morningness in the Baependi population (von Schantz, Taporoski et al., 2015), it is not, however, likely to account for a large proportion of it. Studies in populations with higher, and more well-defined contributions of Amerindian ancestry would be needed to explore this aspect further in 
Egan et al: Association between ancestry and MEQ score

addition to studies investigating the biological variables underlying the MEQ score, and their possible relevance to health disparities between different racial/ethnic groups.

\section{Declaration of interest statement}

The authors have no conflicts of interest to declare. 
Egan et al: Association between ancestry and MEQ score

\section{Acknowledgments}

This study was supported by awards from CNPq to HV and MvS (400791/2015-5), and by the Global Innovation Initiative to MvS (jointly funded by the British Council and the UK Department of Business and Skills). The data collection was supported by Fundação de Amparo à Pesquisa do Estado de São Paulo (FAPESP 2012/05447-0; 2012/12042-7; 2013-17368-0), and PROADI_SUS (25000.180664/2011-35) 


\title{
References
}

\author{
Alexander DH, Novembre J, Lange K. (2009). Fast model-based estimation of ancestry \\ in unrelated individuals. Genome Research. 19:1655-1664. \\ Benedito-Silva, A. A., Menna-Barreto, L., Marques, N. \& Tenreiro, S (1989). in XIX. \\ International Conference of the International Society for Chronobiology. (eds \\ Hayes, D. K., Pauly, J. E. \& Reiter, R. J.) 89-98 (Alan R. Liss, Inc). \\ Dall'Ara I, Ghirotto S, Ingusci S, Bagarolo G, Bertolucci C, Barbujani G. (2016). \\ Demographic history and adaptation account for clock gene diversity in humans. \\ Heredity.
}

de Andrade M, Amos Cl, Thiel TJ. (1999). Methods to estimate genetic components of variance for quantitative traits in family studies. Genetic Epidemiology. 17:64-76.

Eastman CI, Suh C, Tomaka VA, Crowley SJ. (2015). Circadian rhythm phase shifts and endogenous free-running circadian period differ between African-Americans and European-Americans. Scientific Reports (UK). 5:8381.

Egan KJ, von Schantz M, Negrão AB, Horimoto ARVR, Soler JM, de Andrade M, Lorenzi-Filho G, Vallada H, Taporoski TP, Pedrazzoli M, Azambuja A, de Oliveira CM, Alvim RO, Krieger JE, Pereira AC. (2016). Cohort profile: The Baependi Heart Study — a family-based, highly admixed cohort study in a rural Brazilian town. BMJ Open 2016;6:e011598.

Halder I, Matthews KA, Buysse DJ, Strollo PJ, Causer V, Reis SE, Hall MH. (2015). African Genetic Ancestry is Associated with Sleep Depth in Older African Americans. Sleep. 38:1185-1193. 
Horne JA, Östberg O. (1976). A self-assessment questionnaire to determine morningness-eveningness in human circadian rhythms. International journal of chronobiology. 4:97-110.

Hut RA, Paolucci S, Dor R, Kyriacou CP, Daan S. (2013). Latitudinal clines: an evolutionary view on biological rhythms. Proceedings Biological sciences / The Royal Society. 280:20130433.

Nadkarni NA, Weale ME, von Schantz M, Thomas MG. (2005). Evolution of a length polymorphism in the human PER3 gene, a component of the circadian system. Journal of Biol Rhythms. 20:490-499

von Schantz M, Taporoski TP, Horimoto ARVR, Duarte NE, Vallada H, Krieger JE, Pedrazzoli M, Negrão AB, Pereira AC. (2015). Distribution and heritability of diurnal preference (chronotype) in a rural Brazilian family-based cohort, the Baependi study. Scientific Reports (UK). 5:9214. 


\title{
Figure and Table legends
}

Figure 1: (A) Triplot summarizing the Amerindian (AMR), African (AFR) and European (EUR) ancestral contributions within the Baependi population. (B) shows a scatterplot of percentage Amerindian ancestry and MEQ score, with a regression line representing unadjusted data.

\begin{abstract}
Table 1: The relationship between ancestry and diurnal preference explored through eight separate polygenic mixed model regression models. Models 1, 2, and 3 include estimates for European (EUR), African (AFR) and Amerindian (AMR) ancestry, respectively. Models 4-8 explore the significance of AMR results in more detail, adjusting for sex, age, residential zone, and education.
\end{abstract}


Egan et al: Association between ancestry and MEQ score

\begin{tabular}{|c|c|c|c|c|}
\hline Model & Covariate & $\beta$ (effect) & $\begin{array}{l}\text { Standard } \\
\text { error }\end{array}$ & $p$ value \\
\hline 1. EUR & EUR & 0.021 & 0.018 & 0.25 \\
\hline 2. AFR & AFR & -0.034 & 0.018 & 0.056 \\
\hline 3. AMR & AMR & 0.22 & 0.070 & 0.0015 \\
\hline \multirow[t]{2}{*}{ 4. Sex, AMR } & Sex & -1.74 & 0.57 & 0.0023 \\
\hline & AMR & 0.219 & 0.069 & 0.0015 \\
\hline \multirow[t]{2}{*}{ 5. Age, AMR } & Age & 0.319 & 0.015 & $<0.00001$ \\
\hline & AMR & 0.214 & 0.063 & 0.0012 \\
\hline \multirow[t]{2}{*}{ 6. Residential zone, AMR } & Residential zone & 6.156 & 0.855 & $6.1 \times \mathrm{e}^{-13}$ \\
\hline & AMR & 0.225 & 0.066 & $6.7 \times \mathrm{e}^{-04}$ \\
\hline \multirow[t]{3}{*}{ 7. Sex, age, AMR } & Sex & -1.54 & 0.499 & 0.0021 \\
\hline & Age & 0.318 & 0.015 & $<0.00001$ \\
\hline & AMR & 0.215 & 0.066 & 0.0012 \\
\hline \multirow{5}{*}{$\begin{array}{l}\text { 8. Sex, age, residential } \\
\text { zone, education, AMR }\end{array}$} & Sex & -1.27 & 0.489 & $9.3 \times \mathrm{e}^{-03}$ \\
\hline & Age & 0.263 & 0.0173 & $<0.00001$ \\
\hline & Residential zone & 4.75 & 0.780 & $1.2 \times \mathrm{e}^{-09}$ \\
\hline & Education & -0.590 & 0.112 & $1.2 \times \mathrm{e}^{-07}$ \\
\hline & AMR & 0.1624 & 0.062 & $8.8 \times \mathrm{e}^{-03}$ \\
\hline
\end{tabular}


(A)

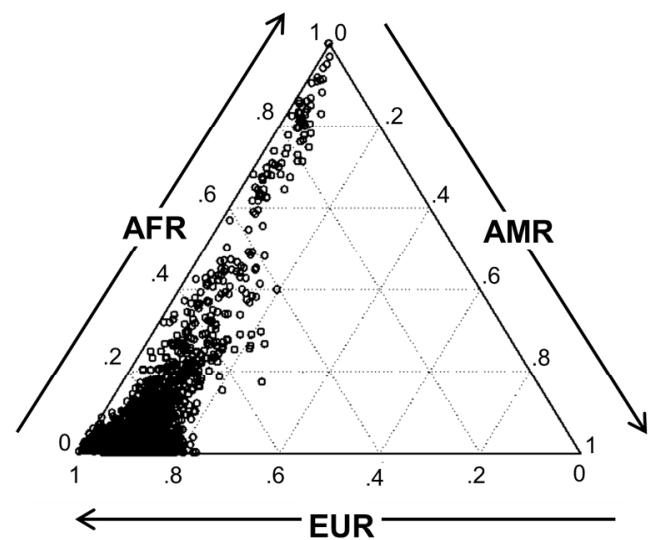

(B)

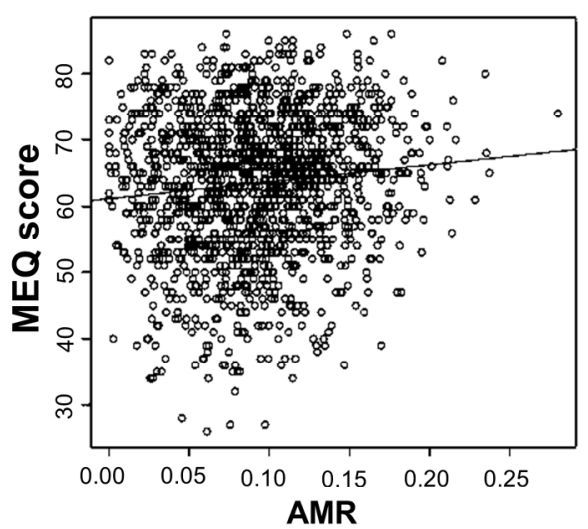

(A) Triplot summarizing the Amerindian (AMR), African (AFR) and European (EUR) ancestral contributions within the Baependi population. (B) shows a scatterplot of percentage Amerindian ancestry and MEQ score, with a regression line representing unadjusted data.

$175 \times 99 \mathrm{~mm}(300 \times 300 \mathrm{DPI})$ 